tion of Sirt 1 as a protectant against other clinically relevant renal insults, and an investigative path that would culminate, hopefully, in the discovery of novel therapies for human kidney disease.

\section{Acknowledgments}

This work is supported by NIH grants R01 DK47060 and HL55552.

Address correspondence to: Karl A. Nath, Mayo Clinic, Guggenheim 542, 200 First St., SW, Rochester, MN 55905. Phone: 507.284.1646; Fax: 507.284.3757; E-mail: nath.karl@mayo.edu.

1. Schoolwerth A, Drewnowska K. Renal Metabolism. In: Schrier R, Gottschalk C, eds. Diseases of the Kidney. New York, NY: Little, Brown and Company; 1997:203-230.

2. Balaban RS, Nemoto S, Finkel T. Mitochondria, oxidants, and aging. Cell. 2005;120(4):483-495.

3. Kume S, et al. Calorie restriction enhances cell adaptation to hypoxia through SIRT1-dependent mitochondrial autophagy in mouse aged kidney. J Clin Invest. 2010;120(4):1043-1055.

4. Tanaka $T$, et al. Hypoxia and expression of hypoxia-inducible factor in the aging kidney. J Gerontol $A$ Biol Sci Med Sci. 2006;61(8):795-805.

5. Chandel NS, Budinger GR. The cellular basis for diverse responses to oxygen. Free Radic Biol Med. 2007;42(2):165-174.

6. Hiromura K, Pippin JW, Fero ML, Roberts JM, Shankland SJ. Modulation of apoptosis by the cyclindependent kinase inhibitor p27(Kip1). J Clin Invest. 1999;103(5):597-604.

7. Dioum EM, et al. Regulation of hypoxia-inducible factor 2alpha signaling by the stress-responsive deacetylase sirtuin 1. Science. 2009;324(5932):1289-1293.

8. Guarente L. Cell biology. Hypoxic hookup. Science. 2009;324(5932):1281-1282.

9. Fine LG, Norman JT. Chronic hypoxia as a mechanism of progression of chronic kidney diseases: from hypothesis to novel therapeutics. Kidney Int. 2008;74(7):867-872.

10. Haase VH. Oxygen regulates epithelial-to-mesenchymal transition: insights into molecular mechanisms and relevance to disease. Kidney Int. 2009; 76(5):492-499.

11. Tanaka T, Nangaku M. The role of hypoxia, increased oxygen consumption, and hypoxia-inducible factor-1 alpha in progression of chronic kidney disease. Curr
Opin Nephrol Hypertens. 2010;19(1):43-50.

12. Neuhofer W, Beck FX. Survival in hostile environments: strategies of renal medullary cells. Physiology (Bethesda). 2006;21(1-4):171-180.

13. Burg MB, Ferraris JD, Dmitrieva NI. Cellular response to hyperosmotic stresses. Physiol Rev. 2007; 87(4):1441-1474.

14. Cowley AW Jr. Renal medullary oxidative stress, pressure-natriuresis, and hypertension. Hypertension. 2008;52(5):777-786.

15 . He W, et al. Sirt 1 activation protects the mouse renal medulla from oxidative injury. J Clin Invest. 2010;120(4):1056-1068

16. Hao CM, Breyer MD. Physiological regulation of prostaglandins in the kidney. Annu Rev Physiol. 2008;70:357-377.

17. Rosenthal T, Shamiss A, Holtzman E. Dietary electrolytes and hypertension in the elderly. Int Urol Nephrol. 2001;33(3):575-582.

18. Nath KA. Tubulointerstitial changes as a major determinant in the progression of renal damage. Am J Kidney Dis. 1992;20(1):1-17.

19. Nath KA. Heme oxygenase-1: a provenance for cytoprotective pathways in the kidney and other tissues. Kidney Int. 2006;70(3):432-443.

20. Schmitt R, Cantley LG. The impact of aging on kidney repair. Am J Physiol Renal Physiol. 2008; 294(6):F1265-F1272.

\title{
The one-two punch: knocking out angiotensin II in the heart
}

\section{Daniela Zablocki and Junichi Sadoshima}

Department of Cell Biology and Molecular Medicine, Cardiovascular Research Institute, University of Medicine and Dentistry of New Jersey, New Jersey Medical School, Newark, New Jersey.

\begin{abstract}
Ang II plays an important role in the pathophysiology of cardiovascular disease. Angiotensin-converting enzyme (ACE) inhibitors lower Ang II levels by inhibiting conversion of Ang I to Ang II, but Ang II levels have been shown to return to normal with chronic ACE inhibitor treatment. In this issue of the JCI, Wei et al. show that ACE inhibition induces an increase in chymase activity in cardiac interstitial fluid, providing an alternate pathway for Ang II generation.
\end{abstract}

Despite ongoing advances in modern medicine, cardiovascular disease (CVD) remains a major cause of death. Ang II has been implicated in the pathophysiology of atherosclerosis and heart failure (HF) due to its role in regulating multiple renal and cardiovascular functions, including salt/water retention, vasoconstriction, aldosterone secretion, cardiac hypertrophy, thrombosis, fibrosis, and others (1). In the classical renin-angiotensin system (RAS), angiotensinogen (AGT) produced in the liver enters the circulation,

Conflict of interest: The authors have declared that no conflict of interest exists.

Citation for this article: J Clin Invest. 2010; 120(4):1028-1031. doi:10.1172/JCI42644. where it is cleaved by renin produced in the kidneys to form Ang I. Ang I is converted to Ang II by angiotensin-converting enzyme (ACE) bound to vascular endothelial cells. Ang II then binds to either the Ang II type 1 receptor (AT1), through which it exerts most of its known effects, or to the Ang II type 2 receptor (AT2), which is thought to oppose AT1 (2). However, research conducted over the last few decades has shown that the RAS is much more complex in both mechanism and effect than once thought (Figure 1).

Some of this complexity comes from the discovery of nonclassical RAS components. Although Ang II is generally considered to be the main RAS effector molecule, other Ang peptides have been discovered, including
Ang-(1-7), Ang-(1-9), Ang-(2-8), Ang-(3-8), and Ang-(1-12) (2,3). In particular, Ang-(1-7) opposes the effects of Ang II through binding to the Mas receptor (3) and inhibits bradykinin (BK) degradation, possibly through competitive binding to ACE (4), while Ang-(1-12) is an alternative precursor for Ang I and II generation (3). In addition, other enzymes cleave AGT and its products. Cathepsin, like renin, generates Ang I from AGT (5), ACE2, an ACE homolog, and neutral endopeptidases cleave Ang I and II to form Ang-(1-7) (2, 6), and some chymases efficiently cleave Ang I to produce Ang II (7) and may be responsible for generation of Ang II from Ang-(1-12) (3). Furthermore, both ACE and chymase have additional functions besides Ang peptide cleavage. ACE is also a kininase (8), linking the RAS and the kallikrein/kinin system, while chymase activates procollagenase and MMPs and catalyzes degradation of thrombin and plasmin, playing an important role in tissue remodeling (7).

How and when these various components come into play depends on the context. 


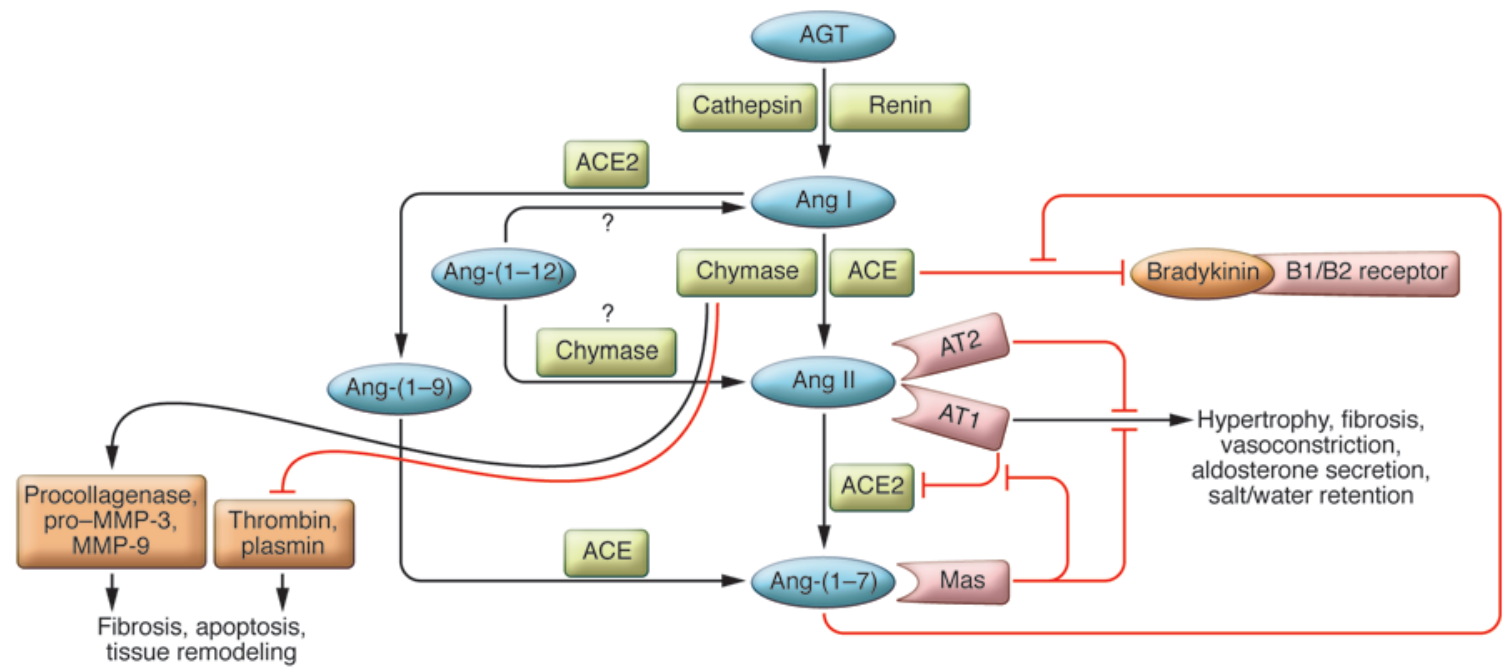

\section{Figure 1}

The renin-angiotensin system. AGT is cleaved by either renin or cathepsin to form Ang I. Ang I is cleaved by ACE or chymase to form Ang II or by ACE2 to form Ang-(1-9). Ang II binds to one of the Ang II receptors to exert its downstream effects on cardiovascular function, with AT1 and AT2 working in opposition to each other. Ang-(1-12) is an alternative precursor for Ang I and II. Ang-(1-7) is formed through cleavage of Ang II by ACE2 or cleavage of Ang-(1-9) by ACE and binds to the Mas receptor. The ACE-Ang II-AT1 axis and the ACE2-Ang-(1-7)-Mas axis appear to have opposing functions. ACE also degrades BK, serving as a link between the RAS and the kallikrein/kinin system, while chymase is also important in tissue remodeling.

Although the RAS was originally thought to function only as a circulatory system, it has since been established that local intracellular and extracellular RAS exist in several tissues, including the heart, kidneys, brain, VSMCs, and BM-derived cells $(2,5,9)$. Ang II generated by tissue RAS may function intracellularly to exert an intracrine effect on local RAS function or may contribute to extracellular Ang II levels to exert an autocrine/paracrine effect, depending on the cell type and conditions $(2,5,9)$.

\section{ACE inhibition increases cardiac interstitial chymase activity}

CVD is highly associated with RAS upregulation. In particular, HF in humans is associated with increased ACE transcription and activity in proportion to disease severity (7). ACE inhibitors (ACEIs) have therefore become a major component of standard therapy for CVD (10). However, although acute ACE inhibition decreases plasma Ang II levels, an escape phenomenon occurs with chronic treatment, with Ang II returning to normal levels (11). Furthermore, the specific mechanisms and relative contributions of the systemic and local RAS in the heart are not well understood. In this issue of the JCI, Wei et al. (12) provide additional insight into the RAS of the cardiac interstitium, the environment in which cardiac myocytes reside.
Previous studies in dogs showed that Ang II generation/degradation in the heart is compartmentalized (13) and that both ACE and chymase are important for Ang II generation in the cardiac interstitial fluid (ISF) (14). In addition, infusion of Ang I or Ang-(1-7) into the ISF was shown to increase BK levels, possibly due to inhibition of ACE (4).

In their current study (12), Wei et al. used ACE selective [Pro $\left.{ }^{10}\right]$ Ang I, and chymase selective $\left[\mathrm{Pro}^{11}\right.$,DAla $\left.{ }^{12}\right]$ Ang I to determine the relative contributions of ACE and chymase to basal ISF Ang II generation. Interestingly, although there was abundant non-ACE activity in LV homogenates, in agreement with previous reports that chymase is a major cardiac Ang II-forming enzyme (14, $15)$, microdialysis probe measurements with these enzyme selective substrates indicated that basal chymase-like activity in the ISF of conscious mice was negligible (12).

Subsequent experiments demonstrated that ACEI treatment for two weeks inhibited ACE activity but did not significantly decrease ISF Ang II levels (12). Wei et al. then showed that chronic ACE inhibition increased transcription and activity of MMCP4, an Ang II-forming chymase, and that ISF Ang II levels decreased following dual inhibition of ACE and MMCP4 (12). Experiments with the kinin B2 receptor (B2R) antagonist, Hoe-140, revealed that the ACEI-induced increase in
ISF chymase activity and subsequent Ang II formation was dependent on B2R activation (12). Thus, it appears that chronic ACE inhibition increases $\mathrm{BK}$, which causes an increase in chymase activity and maintenance of Ang II levels in the mouse LV ISF (Figure 2). These results are somewhat surprising, as it has been suggested that BK may mediate the beneficial effects of ACEIs. However, excess BK is proinflammatory (8), which could explain its apparently detrimental role here.

\section{Possible benefits of dual therapy}

Although ACEI therapy clearly improves outcomes in HF patients $(16,17)$, mortality and reduced quality of life due to HF and CVD remain high. Current guidelines recommend combining ACEIs with AT1 blockers (ARBs) (10) under the working assumption that ACEIs inhibit some Ang II generation and beneficially increase BK levels, while ARBs prevent downstream signaling by remaining Ang II. Nevertheless, this mode of dual therapy remains controversial. Both the Valsartan-Heart Failure Trial (Val-HeFT) and the Candesartan in Heart Failure: Assessment of Reduction in Mortality and Morbidity-Added (CHARM-Added) Trial found a slight improvement in BP and a significant improvement in mortality and morbidity due to congestive HF or CVD in patients treated with both ACEIs and ARBs compared with those treated with ACEIs 


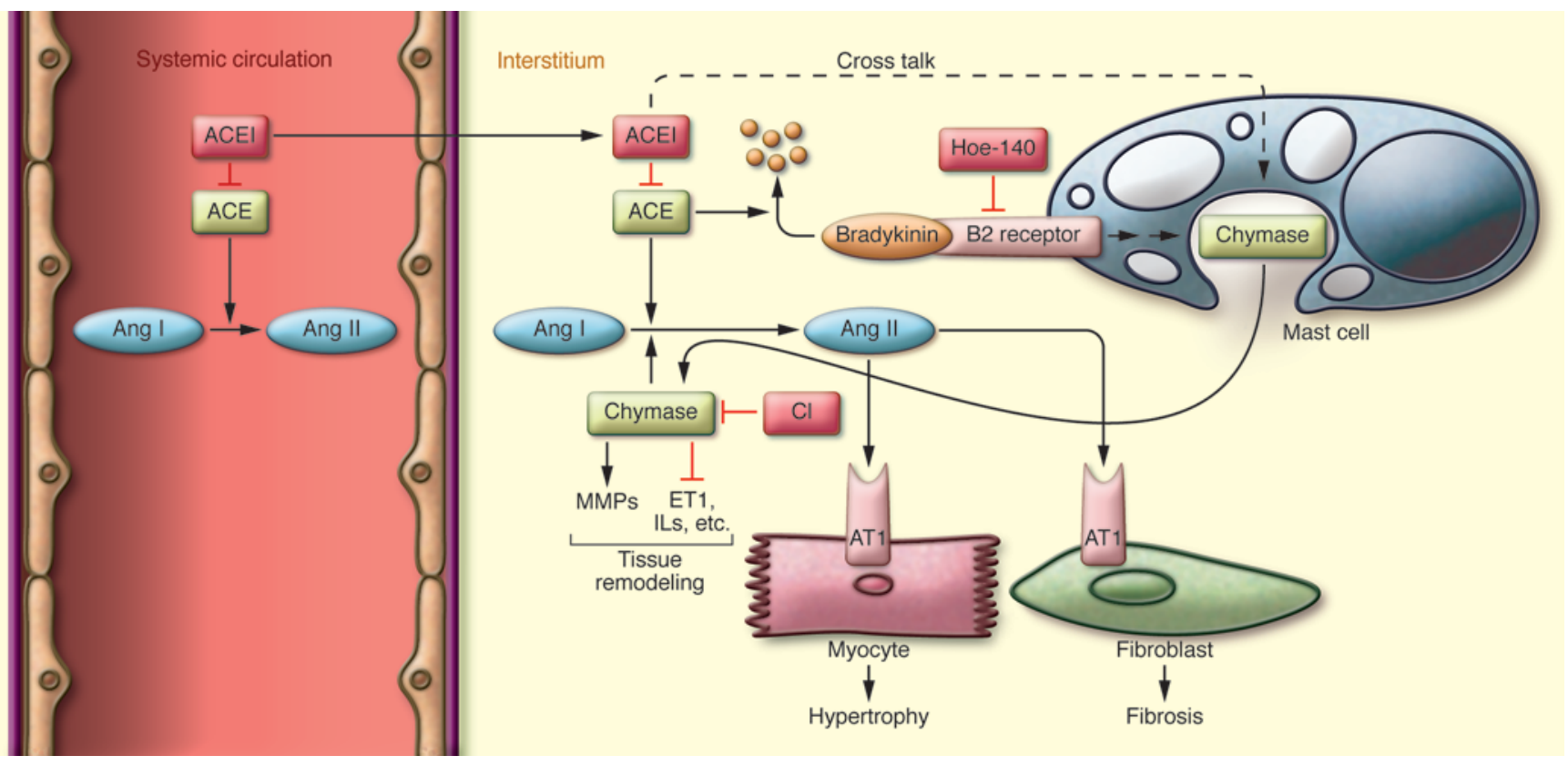

Figure 2

ACE inhibition induces chymase release, which prevents decreased Ang II formation. ACEl enters the interstitium from the systemic circulation and inhibits degradation of BK by ACE. Increased BK signaling through the B2R leads to chymase release, creating an alternative pathway for Ang II generation and contributing to adverse cardiac tissue remodeling. Inhibition of B2R signaling by Hoe-140 prevents ACEl-induced chymase release and suppresses Ang II formation. Similarly, LV function is improved by inhibition of chymase in conjunction with ACEI.

alone $(18,19)$. However, dual therapy did not significantly improve overall mortality. On the other hand, in the Ongoing Telmisartan Alone and in Combination with Ramipril Global Endpoint Trial (ONTARGET), dual therapy caused worse renal outcomes than either ACEI or ARB alone (20), suggesting that the risks may outweigh the benefits.

To characterize the physiological relevance of chronic ACEI-induced chymase release, Wei et al. examined the effects of ACEI, chymase inhibitor, and combination treatment following myocardial infarction (MI) in hamsters (12). They found that dual treatment led to significant improvements in LV function and remodeling. Perhaps most striking is that survival in this group was comparable to that of the sham-operated group. These results suggest that dual ACEI/chymase inhibitor treatment may represent a viable alternative method for blocking Ang II function.

\section{Remaining questions}

Some questions remain regarding the mechanism of chymase induction and Ang II maintenance. Wei et al. suggest that the ACEI-induced increase in ISF chymase activity is due to degranulation of mast cells (MCs) by BK. As evidence of this, they cite the presence of $\mathrm{B} 2 \mathrm{R} / \mathrm{MMCP} 4$-positive
MCs in the LV and absence of detectable chymase mRNA or expression in MCdeficient $K i t^{W} / K i t^{W-v}$ mice (12). However, because of technical difficulties, they were unable to definitively prove that MCs, not other c-kit-expressing cells, were responsible for the chymase activity. Nor could they determine whether BK affects chymase levels directly, by degranulating MCs, or indirectly, perhaps by increasing $\mathrm{MC}$ recruitment. In the functional study, although the authors describe phenotypic changes that occur in the different treatment groups, there is no data on post-MI Ang II levels. It therefore remains to be determined whether the benefits of dual inhibition are caused by reduced Ang II generation or suppression of chymase-associated LV remodeling. Furthermore, the study addresses only the extracellular RAS in the cardiac ISF. However, the intracellular RAS of cardiac myocytes and fibroblasts (which are themselves differentially regulated; see ref. 5) are likely also significant in determining the post-MI phenotype. Resolution of these mechanistic issues will be important in determining how and when chymase inhibition might be appropriate in a therapeutic setting.

Another important issue is the effect of interspecies differences in RAS components and function. There is tremendous variation in both the numbers and types of chymases, which may be Ang II forming and/or Ang II degrading (7). The relative contributions of ACE and chymase to Ang II formation also vary among species, as does Ang II sensitivity and signaling. Because of this, Wei et al. used hamsters, in which cardiac myocyte Ang II sensitivity more closely resembles that of humans, for their functional studies (12). Nevertheless, caution must be exercised in translating these results into the context of the human RAS.

Perhaps the most important question raised by this study, however, concerns the accepted rationale for ACEI treatment, namely suppression of Ang II. Given the existence of the escape phenomenon, it has been suggested that increased BK signaling, rather than reduced Ang II, may be responsible for the continuing beneficial effects of chronic ACE inhibition. However, if BK causes MC recruitment/degranulation and chymase release, as this study suggests, the increased BK may be at least partially responsible for maintained Ang II levels and may actually be detrimental rather than beneficial. Alternatively, ACE inhibition increases ACE2 expression, and the ACE2-Ang-(1-7)-Mas receptor axis has been shown to oppose the functions of the ACE-Ang II-AT1 axis $(2,3,6)$, pro- 
viding another possible mechanism for the effectiveness of chronic ACE inhibition. Also, although the current study focuses primarily on the effect of chymase release on Ang II levels, chymase contributions to adverse LV remodeling may possibly be more important. If Ang II is not actually the main effector molecule, a better understanding of how ACEIs exert their effects could lead to further improvements in therapeutic outcomes.

\section{Acknowledgments}

This work was supported in part by NIH grants R01 HL67724, R01 AG023039, R01 HL091469, P01 HL69020, and P01 AG027211 and the Foundation Leducq Transatlantic Network of Excellence.

Address correspondence to: Junichi Sadoshima, Cardiovascular Research Institute, UMDNJ - Newark, 185 S. Orange Ave., MSB G609, Newark, New Jersey 07103. Phone: 973.972.8619; Fax: 973.972.8919; E-mail: sadoshju@umdnj.edu.

1. Nicholls MG, Robertson JI, Inagami T. The reninangiotensin system in the twenty-first century. Blood Press. 2001;10(5-6):327-343.

2. Kurdi M, De Mello WC, Booz GW. Working outside the system: an update on the unconventional behavior of the renin-angiotensin system components. Int J Biochem Cell Biol. 2005;37(7):1357-1367.

3. Ferrario CM. New physiological concepts of the renin-angiotensin system from the investigation of precursors and products of angiotensin I metabolism. Hypertension. 2010;55(2):445-452.

4. Wei CC, et al. Angiotensin peptides modulate bradykinin levels in the interstitium of the dog heart in vivo. J Pharmacol Exp Ther. 2002;300(1):324-329.

5. Kumar R, Singh VP, Baker KM. The intracellular renin-angiotensin system: implications in cardiovascular remodeling. Curr Opin Nephrol Hypertens. 2008; 17(2):168-173.

6. Crackower MA, et al. Angiotensin-converting enzyme 2 is an essential regulator of heart function. Nature. 2002;417(6891):822-828.

7. Dell'Italia LJ, Husain A. Dissecting the role of chymase in angiotensin II formation and heart and blood vessel diseases. Curr Opin Cardiol. 2002; 17(4):374-379.

8. Kaplan AP, Joseph K, Silverberg M. Pathways for bradykinin formation and inflammatory disease. J Allergy Clin Immunol. 2002;109(2):195-209.

9. Bader M, Ganten D. Update on tissue renin-angiotensin systems. J Mol Med. 2008;86(6):615-621.

10. Dickstein K, et al. ESC guidelines for the diagnosis and treatment of acute and chronic heart failure 2008: the Task Force for the diagnosis and treatment of acute and chronic heart failure 2008 of the European Society of Cardiology. Developed in collaboration with the Heart Failure Association of the ESC (HFA) and endorsed by the European Society of Intensive Care Medicine (ESICM). EurJ Heart Fail. 2008;10(10):933-989.

11. Biollaz J, Brunner HR, Gavras I, Waeber B, Gavras H. Antihypertensive therapy with MK 421: angiotensin II--renin relationships to evaluate efficacy of converting enzyme blockade. J Cardiovasc Pharmacol. 1982;4(6):966-972.
12. Wei C-C, et al. Mast cell chymase limits the cardiac efficacy of Ang I-converting enzyme inhibitor therapy in rodents. J Clin Invest. 2010;120(4):1229-1239.

13. Dell'Italia LJ, et al. Compartmentalization of angiotensin II generation in the dog heart. Evidence for independent mechanisms in intravascular and interstitial spaces. J Clin Invest. 1997;100(2):253-258.

14. Wei CC, et al. Evidence for angiotensin-converting enzyme- and chymase-mediated angiotensin II formation in the interstitial fluid space of the dog heart in vivo. Circulation. 1999;99(19):2583-2589.

15. Urata H, Kinoshita A, Misono KS, Bumpus FM, Husain A. Identification of a highly specific chymase as the major angiotensin II-forming enzyme in the human heart. J Biol Chem. 1990; 265(36):22348-22357.

16. The CONSENSUS Trial Study Group. Effects of enalapril on mortality in severe congestive heart failure. Results of the Cooperative North Scandinavian Enalapril Survival Study (CONSENSUS). NEngl J Med. 1987;316(23):1429-1435.

17. The SOLVD Investigators. Effect of enalapril on survival in patients with reduced left ventricular ejection fractions and congestive heart failure. N Engl J Med. 1991;325(5):293-302.

18. Krum H, et al. Effect of valsartan added to background ACE inhibitor therapy in patients with heart failure: results from Val-HeFT. Eur J Heart Fail. 2004;6(7):937-945.

19. McMurray JJ, et al. Effects of candesartan in patients with chronic heart failure and reduced left-ventricular systolic function taking angiotensin-convertingenzyme inhibitors: the CHARM-Added trial. Lancet. 2003;362(9386):767-771.

20. Mann JF, et al. Renal outcomes with telmisartan, ramipril, or both, in people at high vascular risk (the ONTARGET study): a multicentre, randomised, double-blind, controlled trial. Lancet. 2008; 372(9638):547-553

\title{
Epithelial-mesenchymal transitions and hepatocarcinogenesis
}

\author{
Janice Jou and Anna Mae Diehl
}

Division of Gastroenterology, Duke University, Durham, North Carolina.

\begin{abstract}
Epithelial-mesenchymal transitions (EMTs) are believed to play a role in invasion and metastasis of many types of tumors. In this issue of the JCI, Chen et al. show that a gene that has been associated with aggressive biology in hepatocellular carcinomas initiates a molecular cascade that results in EMT.
\end{abstract}

Hepatocellular carcinoma (HCC) is the fifth most commonly diagnosed, and the third most deadly, cancer worldwide (1). In the United States between 1975 and 2006, HCC was the only cancer with increasing mortality in men and women (2). Surveillance in patients at increased risk for developing HCC has been recommended for

Conflict of interest: The authors have declared that no conflict of interest exists.

Citation for this article: J Clin Invest. 2010; 120(4):1031-1034. doi:10.1172/JCI42615. detection of early HCC (3); however, this is underutilized, and many patients present with locally advanced or metastatic disease. These patients are not liver transplantation candidates and have limited therapeutic options. Thus, there is an urgent need for identification of patients at risk for HCC and further risk stratification of these patients to improve the outcomes. Therefore, groups like Chen et al. (4) are applying gene profiling to identify better prognostic and/or therapeutic targets. Their present work, in this issue of the JCI, reveals that the oncogene chromodomain helicase/ATPase DNA binding protein 1-like gene $(C D H 1 L)$ is commonly amplified in HCC (4).

Chen et al. demonstrated why CDH1L amplification is important in HCC (Figure 1 and ref. 4). CDH1L functions as a transcription factor that induces expression of the guanine nucleotide exchange factor (GEF) ARHGEF9. GEFs catalyze exchange of GDP for GTP on RhoGTPases, a family of GTPases that includes Rho and Rac proteins as well as Cdc42 (5). Chen et al. demonstrated that Cdc42 was the target of ARHGEF9 and that increased ARHGEF9 enhanced formation of Cdc42-GTP (i.e., activated Cdc42) in HCC cells (4). Cdc42 collaborates with other Rho kinases to modulate assembly and disassembly of the actin cytoskeleton 